\title{
NILPOTENT LIE ALGEBRAS HAVING THE SCHUR MULTIPLIER OF MAXIMUM DIMENSION
}

\author{
AFSANEH SHAMSAKI AND PEYMAN NIROOMAND
}

\begin{abstract}
Let $L$ be an $n$-dimensional nilpotent Lie algebra of nilpotency class $c$ with the derived subalgebra of dimension $m$. Recently, Rai proved that the dimension of Schur multiplier of $L$ is bounded by $\frac{1}{2}(n-m-1)(n+m)-$ $\min \{n-m, c\}$

$\sum_{i=2} n-m-i$. In this paper, we obtain the structure of all nilpotent

Lie algebras that attain this bound.
\end{abstract}

\section{INTRODUCTION}

The Schur multiplier of groups was introduced by Schur in [14 on the study of projective representation of groups. It is known from [7, Main Theorem] that for a non-abelian $p$-group $G$ of order $p^{n}$ and the derived subgroup of order $p^{k}$, we have

$$
|\mathcal{M}(G)| \leq p^{\frac{1}{2}(n-k-1)(n+k-2)+1} .
$$

Recently Rai in [11, Theorem 1.1] classified $p$-groups of nilpotency class two such that $|\mathcal{M}(G)|=p^{\frac{1}{2}(n-k-1)(n+k-2)+1}$ and then Hatui in [2] showed for $p \neq 3$ only nilpotent groups of class 2 satisfy in the upper bound 1.1. By a classical result due to Lazard 4, we may associate a $p$-group to a Lie algebra. Analogous to the Schur multiplier of a group, the Schur multiplier of a Lie algebra, $\mathcal{M}(L)$, can be defined as $\mathcal{M}(L) \cong R \cap F^{2} /[R, F]$ where $L \cong F / R$ and $F$ is a free Lie algebra by the same motivation in [6. Theorem 3.1], the second author proved that for a non-abelian nilpotent Lie algebra of dimension $n$ and the derived algebras of dimension $m$, we showed

$$
\operatorname{dim} \mathcal{M}(L) \leq \frac{1}{2}(n+m-2)(n-m-1)+1 .
$$

In particular, when $m=1$ the bound is attained if and only if $L \cong H(1) \oplus A(n-3)$ where $H(m)$ and $A(n)$ are used to denote the Heisenberg and Abelian Lie algebras of dimension $2 m+1$ and $n$, respectively. Looking [9] shows similar results of Hatui are obtained for the class of nilpotent Lie algebra. Recently, Rai in [12, Theorem 1.1] improved the bound 1.2 and showed

$$
\operatorname{dim} \mathcal{M}(L) \leq \frac{1}{2}(n-m-1)(n+m)-\sum_{i=2}^{\min \{n-m, c\}} n-m-i,
$$

for an $n$-dimensional nilpotent Lie algebra $L$ of nilpotency class $c$ when $\operatorname{dim} L^{2}=$ $m \geq 1$.

In the current paper, we are going to classify all nilpotent Lie algebras that attain the bound 1.3. More precisely, we show that such Lie algebras are nilpotent of class

Key words and phrases. nilpotent Lie algebra. 
two.

\section{Preliminaries}

For the convenience of the reader, we give some results without proofs which will be used in the next section.

We recall two theorems.

Theorem 2.1. (See [6, Corollary 2.3]) Let L be a finite dimensional Lie algebra and $K$ be a central ideal of $L$. Then

$\operatorname{dim} \mathcal{M}(L)+\operatorname{dim}\left(L^{2} \cap K\right) \leq \operatorname{dim} \mathcal{M}(L / K)+\operatorname{dim} \mathcal{M}(K)+\operatorname{dim}\left((L / K)^{a b} \otimes_{a b} K\right)$.

Theorem 2.2. (See [7, Theorem 3.1]) Let L be an n-dimensional nilpotent Lie algebra $L, \operatorname{dim} L^{2}=n-2$ and $n \geq 4$. Then $\operatorname{dim} \mathcal{M}(L) \leq \operatorname{dim} L^{2}$.

Let $Z^{\wedge}(L)$ is used to denote the exterior center of a Lie algebra $L$ (See [5] for more information). By [5, $L$ is capable if and only if $Z^{\wedge}(L)=0$.

Here is easy result.

Lemma 2.3. (See [10, Corollary 2.3]) Let $L$ be a finite dimensional non-abelian nilpotent Lie algebra. Then $Z^{\wedge}(L) \subseteq L^{2}$.

Let $\otimes_{a b}$ denotes the operator of the standard tensor product of Lie algebras (See [1, page 103]). Then

Proposition 2.4. (See [9, Proposition 2.3]) Let L be a Lie algebra. Then

(i) the map $\gamma_{L}: L^{a b} \otimes_{\text {mod }} L^{a b} \otimes_{\text {mod }} L^{a b} \rightarrow L^{2} / L^{3} \otimes_{\text {mod }} L / L^{2}$ given by

$\left(x+L^{2}\right) \otimes\left(y+L^{2}\right) \otimes\left(z+L^{2}\right) \mapsto\left([x, y]+L^{3} \otimes z+L^{2}\right)+\left([z, x]+L^{3} \otimes y+L^{2}\right)+\left([y, z]+L^{3} \otimes x+L^{2}\right)$

is a Lie homomorphism. If any two element of the set $\{x, y, z\} \subseteq L$ are linearly dependent. Then $\gamma_{L}\left(x+L^{2} \otimes y+L^{2} \otimes z+L^{2}\right)=0$.

(ii) Define the map

$$
\begin{aligned}
& \gamma_{2}^{\prime}:(L / Z(L))^{a b} \otimes_{\bmod }(L / Z(L))^{a b} \otimes_{\bmod }(L / Z(L))^{a b} \rightarrow L^{2} / L^{3} \otimes_{\bmod }(L / Z(L))^{a b} \\
& \left(x+\left(L^{2}+Z(L)\right)\right) \otimes\left(y+\left(L^{2}+Z(L)\right)\right) \otimes\left(z+\left(L^{2}+Z(L)\right)\right) \otimes\left(w+\left(L^{2}+Z(L)\right)\right) \mapsto \\
& \left([x, y]+L^{3} \otimes z+\left(L^{2}+Z(L)\right)\right)+\left([z, x]+L^{3} \otimes y+\left(L^{2}+Z(L)\right)\right)+ \\
& \left([y, z]+L^{3} \otimes x+\left(L^{2}+Z(L)\right)\right) .
\end{aligned}
$$

Then $\gamma_{2}^{\prime}$ is a Lie homomorphism. Moreover, if any two element of the set $\{x, y, z\} \subseteq L$ are linearly dependent. Then $\gamma_{2}^{\prime}\left(x+L^{2} \otimes y+L^{2} \otimes z+L^{2}\right)=0$.

(iii) The map $\gamma_{3}^{\prime}:(L / Z(L))^{a b} \otimes_{\text {mod }}(L / Z(L))^{a b} \otimes_{\text {mod }}(L / Z(L))^{a b} \otimes_{\text {mod }}(L / Z(L))^{a b}$

$\rightarrow L^{3} \otimes_{\bmod }(L / Z(L))^{a b}$ given by

$$
\begin{aligned}
& \left(x+\left(L^{2}+Z(L)\right)\right) \otimes\left(y+\left(L^{2}+Z(L)\right)\right) \otimes\left(z+\left(L^{2}+Z(L)\right)\right) \otimes\left(w+\left(L^{2}+Z(L)\right)\right) \\
& \mapsto\left([[x, y], z] \otimes w+\left(L^{2}+Z(L)\right)\right)+\left([w,[x, y]] \otimes z+\left(L^{2}+Z(L)\right)\right)+ \\
& \left([[z, w], x] \otimes y+\left(L^{2}+Z(L)\right)\right)+\left([y,[z, w]] \otimes x+\left(L^{2}+Z(L)\right)\right) .
\end{aligned}
$$

is a Lie homomorphism.

Now there are some results on the dimension of the exterior square of a Lie algebra $L$. 
Theorem 2.5. (See 9, Theorem 2.7]) Let L be a finite dimensional nilpotent nonabelian Lie algebra of class $c$. Then

$$
\begin{aligned}
\operatorname{dim} L \wedge L+\operatorname{dim} \operatorname{Im} \gamma_{2}^{\prime} & \leq \operatorname{dim} L \wedge L+\sum_{i=2}^{c} \operatorname{dim} \operatorname{ker} \alpha_{i} \\
& =\operatorname{dim} L / L^{2} \wedge L / L^{2}+\sum_{i=2}^{c} \operatorname{dim}\left(L^{i} / L^{i+1} \otimes_{\bmod }(L / Z(L))^{a b}\right) .
\end{aligned}
$$

Theorem 2.6. (See [9, Theorem 2.8]) Let L be a finite dimensional nilpotent nonabelian Lie algebra of class 3 . Then, we have

$$
\begin{aligned}
& \operatorname{dim} L \wedge L+\operatorname{dim} \operatorname{Im} \gamma_{2}^{\prime}+\operatorname{dim} \operatorname{Im} \gamma_{3}^{\prime} \\
& \leq \operatorname{dim} L / L^{2} \wedge L / L^{2}+\operatorname{dim}\left(L^{2} / L^{3} \otimes_{\text {mod }} L^{a b}\right)+\operatorname{dim}\left(L^{3} \otimes_{\text {mod }} L^{a b}\right) .
\end{aligned}
$$

Let $Z^{*}(L)$ be a symbol, the epicenter of a Lie algebra $L$. From [5, Lemma 3.1], $Z^{*}(L)=Z^{\wedge}(L)$.

Theorem 2.7. (See [15, Theorem 4.4]) Let $N$ be a central ideal of a Lie algebra $L$. Then the following condition are equivalent.

(i) $\mathcal{M}(L) \cong \mathcal{M}(L / N) / N \cap L^{2}$;

(ii) $N \subseteq Z^{*}(L)=Z^{\wedge}(L)$;

(iii) The natural map $\mathcal{M}(L) \longrightarrow \mathcal{M}(L / N)$ is monomorphism.

Let $\operatorname{cl}(L)$ is used to denote the nilpotency class of $L$.

The following technical results are suitable for the next investigations.

Theorem 2.8. (See [10, Theorem 5.5]) Let $L$ be an $n$-dimensional nilpotent Lie algebra such that $\operatorname{dim} L^{2} \leq 2$. Then $L$ is capable if and only if $L$ is isomorphic to one the following Lie algebras.

(i) If $\operatorname{dim} L^{2}=0$, then $L \cong A(n)$ and $n \geq 2$;

(ii) If $\operatorname{dim} L^{2}=1$, then $L \cong H(1) \oplus A(n-3)$;

(iii) If $\operatorname{dim} L^{2}=2$ and $\operatorname{cl}(L)=2$, then $L \cong L_{6,7}^{2}(\eta) \oplus A(n-6), L \cong L_{5.8} \oplus$ $A(n-5), L \cong L_{6,22}(\varepsilon) \oplus A(n-6)$, or $L \cong L_{1} \oplus A(n-7)$;

(iv) If $\operatorname{dim} L^{2}=2$ and $\operatorname{cl}(L)=3$, then $L \cong L_{4,3} \oplus A(n-4)$, or $L \cong L_{5,5} \oplus$ $A(n-5)$.

Theorem 2.9. (See [13, Theorem 3.2]) There is no Lie algebra L of dimension $n$ such $\operatorname{dim} L^{2}=3$ and $\operatorname{dim} \mathcal{M}(L)=1 / 2(n-1)(n-2)-2$.

Theorem 2.10. (See [9, Theorem 2.22]) Let L be an n-dimensional nilpotent Lie algebra of class two with the drived subalgebra of dimension $m$. Then $\operatorname{dim} \mathcal{M}(L)=$ $\frac{1}{2}(n+m-2)(n-m-1)+1$ if and only if $L$ is isomorphic to one of the Lie algebras $H(1) \oplus A(n-3), L_{5,8}$ or $L_{6,26}$.

\section{MAIN RESULT}

In this section, we classify all nilpotent Lie algebras $L$ such that obtain the upper bound 1.3. Throughout the paper, we say that $\operatorname{dim} \mathcal{M}(L)$ attains the upper bound provided that $\operatorname{dim} \mathcal{M}(L)$ attains the upper bound 1.3 .

Lemma 3.1. There is no n-dimensional nilpotent Lie algebra with the derived subalgebra of dimension $n-2(n \geq 4)$ such that $\operatorname{dim} \mathcal{M}(L)$ attains the bound. 
Proof. Let $\operatorname{dim} \mathcal{M}(L)$ attains the bound then $c \leq n-1$ and $\operatorname{dim} \mathcal{M}(L)=\frac{1}{2}(n-$ $(n-2)-1)(n+(n-2))-\sum_{i=2}^{\min \{2, c\}} n-m-i=n-1$. On the other hand, Theorem 2.2 shows $\operatorname{dim} \mathcal{M}(L) \leq n-2$. It is a contradiction.

Theorem 3.2. Let $L$ be an $n$-dimensional nilpotent Lie algebra of nilpotency class two and $\operatorname{dim} L^{2}=m$. Then $\operatorname{dim} \mathcal{M}(L)$ attains the bound if and only if $L$ is isomorphic to one of the Lie algebras $H(1) \oplus A(n-3), L_{5,8}$ or $L_{6,26}$.

Proof. It is obvious that the bounds 1.2 and 1.3 are equal when $c=2$. Since $\operatorname{dim} \mathcal{M}(L)$ attains the bound and $L$ is of nilpotency class two, $L$ is isomorphic to one of the Lie algebras $H(1) \oplus A(n-3), L_{5,8}$ or $L_{6,26}$ by using Theorem 2.10 ,

Proposition 3.3. Let $L$ be an n-dimensional nilpotent Lie algebra of nilpotency class $c \geq 3$ such that $\operatorname{dim} \mathcal{M}(L)$ attains the bound. If $I$ is an ideal of onedimensional contained in $Z(L) \cap L^{2}$. Then $\operatorname{dim} \mathcal{M}(L / I)$ attains the bound.

Proof. Let $I$ be an ideal of one-dimensional such that contained in $Z(L) \cap L^{2}$, then $\operatorname{dim} L / I=n-1, \operatorname{dim}(L / I)^{2}=m-1$ and $c l(L / I)=c$ or $c-1$.

Let $c l(L / I)=c$. Then by using Theorem 2.1, we have

$$
\begin{aligned}
\operatorname{dim} \mathcal{M}(L)+\operatorname{dim}\left(L^{2} \cap I\right) & \leq \operatorname{dim} \mathcal{M}(L / I)+\operatorname{dim} \mathcal{M}(I)+\operatorname{dim}\left((L / I)^{a b} \otimes I\right) \\
& =\operatorname{dim} \mathcal{M}(L / I)+\operatorname{dim}\left((L)^{a b} \otimes I\right) .
\end{aligned}
$$

Since $c l(L / I)=c,(1.3)$ implies that

$$
\begin{aligned}
\operatorname{dim} \mathcal{M}(L) & \leq \operatorname{dim} \mathcal{M}(L / I)+\operatorname{dim}\left((L)^{a b} \otimes I\right)-\operatorname{dim}\left(L^{2} \cap I\right) \\
& \leq \frac{1}{2}(n-m-1)(n+m-2)-\left(\sum_{i=2}^{\min \{n-m, c\}} n-m-i\right)+(n-m)-1 \\
& =\frac{1}{2}(n-m-1)(n+m)-\sum_{i=2}^{\min \{n-m, c\}} n-m-i=\operatorname{dim} \mathcal{M}(L) .
\end{aligned}
$$

Therefore

$$
\operatorname{dim} \mathcal{M}(L / I)=\frac{1}{2}(n-m-1)(n+m-2)-\sum_{i=2}^{\min \{n-m, c\}} n-m-i .
$$

If $c l(L / I)=c-1$. Then let $n-m \leq c$. So we can see that $\sum_{i=2}^{\min \{n-m, c\}} n-m-i=$ $\min \{n-m, c-1\}$

$\sum_{i=2}^{n-m, c-1\}} n-m-i$. Hence by a similar way to the above case, the result follows.

Suppose that $c l(L / I)=c-1$ and $n-m>c$. Since $c l(L / I)=c-1$, we have $I=\gamma_{c}(L)$ and $\operatorname{dim} \gamma_{c}(L)=1$. On the other hand, by using the proof of [12, Theorem 1.1], we have $\operatorname{dim} \operatorname{ker}\left(\lambda_{c}\right) \geq n-m-c$ and

$$
\operatorname{dim} \mathcal{M}(L)=\operatorname{dim} \mathcal{M}\left(L / \gamma_{c}(L)\right)+\operatorname{dim}\left(L / \gamma_{2}(L)-1\right) \operatorname{dim} \gamma_{c}(L)-\operatorname{dim} \operatorname{ker}\left(\lambda_{c}\right) .
$$


Thus

$$
\begin{aligned}
& \operatorname{dim} \mathcal{M}(L)=\operatorname{dim} \mathcal{M}(L / I)+\left(\operatorname{dim} L / \gamma_{2}(L)-1\right) \operatorname{dim} I-\operatorname{dim} \operatorname{ker}\left(\lambda_{c}\right) \\
& \leq \frac{1}{2}(n-m-1)(n+m-2)-\left(\sum_{i=2}^{c-1} n-m-i\right)+(n-m-1)-(n-m-c) \\
& =\operatorname{dim} \mathcal{M}(L),
\end{aligned}
$$

and so $\operatorname{dim} \mathcal{M}(L / I)=\frac{1}{2}(n-m-1)(n+m-2)-\left(\sum_{i=2}^{\min \{n-m, c\}} n-m-i\right)$.

This completes the proof.

Lemma 3.4. Let $L$ be an $n$-dimensional nilpotent Lie algebra of nilpotency class $c \geq 3$ and $\operatorname{dim} L^{2}=m \geq 2$. If $\operatorname{dim} \mathcal{M}(L)$ attains the bound, then $L$ is capable.

Proof. By contrary, let $L$ be non-capable. Then by using Lemma $2.7 Z^{\wedge}(L) \neq 0$ and Lemma 2.3 shows that there is an ideal $I$ in $Z^{\wedge}(L)$ of dimension one such that $I \subseteq Z(L) \cap L^{2}$. Theorem 2.7 shows that $\operatorname{dim} \mathcal{M}(L)=\operatorname{dim} \mathcal{M}(L / I)-1$. Using Proposition 3.3 we have $\operatorname{dim} \mathcal{M}(L / I)=\frac{1}{2}(n-m-1)(n+m-2)-\left(\sum_{i=2}^{\min \left\{n-m, c^{\prime}\right\}} n-\right.$ $m-i)$, in which $c^{\prime}=c l(L / I)$. Since $c^{\prime}=c$ or $c-1$, we have $\operatorname{dim} \mathcal{M}(L / I)=\frac{1}{2}(n-$ $m-1)(n+m-2)-\left(\sum_{i=2}^{\min \left\{n-m, c^{\prime}\right\}} n-m-i\right)$, and so $\operatorname{dim} \mathcal{M}(L)=\operatorname{dim} \mathcal{M}(L / I)-1=$ $\frac{1}{2}(n-m-1)(n+m-2)-\left(\sum_{i=2}^{\min \{n-m, c\}} n-m-i\right)-1$ or $\frac{1}{2}(n-m-1)(n+m-$ $2)-\left(\sum_{i=2}^{\min \{n-m, c-1\}} n-m-i\right)$. It is a contradiction.

Lemma 3.5. There is no $n$-dimensional nilpotent Lie algebra $L$ of nilpotency class 3 such that $\operatorname{dim} \mathcal{M}(L)$ attains the bound and $\operatorname{cl}(L / I)=2$ for a given onedimensional ideal $I$ in $Z(L) \cap L^{2}$.

Proof. By contrary, let $L$ be an $n$-dimensional nilpotent Lie algebra of nilpotency class 3 such that $\operatorname{dim} \mathcal{M}(L)$ attains the bound. Let $\operatorname{cl}(L / I)=2$ for a given onedimensional ideal $I$ in $Z(L) \cap L^{2}$. By Proposition 3.3. $\operatorname{dim} \mathcal{M}(L / I)$ also attains the bound. Hence by Theorem [3.2, $L / I$ is isomorphic to one of the Lie algebras $H(1) \oplus A(n-3), L_{5,8}$ or $L_{6,26}$. Now we consider all cases. Let $\operatorname{dim} L^{2}=m$. Clearly $m \geq 2$.

Case $(i)$. Here $L / I \cong H(1) \oplus A(n-4)$, then $m=2$. Since $\operatorname{cl}(L)=3$ and $\operatorname{dim} \mathcal{M}(L)$ attains the bound. $L$ is capable by using Lemma 3.4 Now $L$ is isomorphic to one of the Lie algebras $L \cong L_{4,3} \oplus A(n-4)$ or $L_{5,5} \oplus A(n-5)$ by using Theorem 2.8 ,

Let $L \cong L_{4,3} \oplus A(n-4)$, we have $\operatorname{dim} \mathcal{M}(L)=2+\frac{1}{2}(n-4)(n-5)+2(n-4)$. Since $\operatorname{dim} \mathcal{M}(L)$ attains the bound, we have

$$
\frac{1}{2}(n-3)(n+2)-(2 n-9)=2+\frac{1}{2}(n-4)(n-5)+2(n-4),
$$

which is a contradiction.

Let now $L \cong L_{5,5} \oplus A(n-5)$ by a similar way to the above case, we get a contradiction again.

Case $(i i)$. Let $L / I \cong L_{5,8}$, then $n=6, m=3$ and $\operatorname{dim} \mathcal{M}(L)=8=1 / 2(6-1)(6-$ 
$2)-2$. It is a contradiction by looking Theorem 2.9.

Case (iii). Let $L / I \cong L_{6,26}$ and $L$ be minimally generated by $d$ elements. Then $d=3$ and $\operatorname{dim} \mathcal{M}(L)=10$. If $Z(L) \subseteq L^{2}$, then we can choose a basis $B=$ $\left\{x_{1}+L^{2}, x_{2}+L^{2}, x_{3}+L^{2}\right\}$ for $L^{a b}$. We may assume that $\left[x_{1}, x_{2}\right]+L^{3}$ is non-trivial in $L^{2} / L^{3}$. Since $\left[x_{1}, x_{2}\right] \notin L^{3}$ and $x_{2} \notin L^{2}, \gamma_{2}^{\prime}\left(x_{1}+L^{2} \otimes x_{2}+L^{2} \otimes x_{3}+L^{2}\right) \neq 0$. Thus $\operatorname{dim} \operatorname{Im} \gamma_{2}^{\prime} \geq 1$. Let $y \in L \backslash L^{2}$, then $\gamma_{3}^{\prime}\left(x_{1}+L^{2} \otimes x_{2}+L^{2} \otimes x_{3}+L^{2}+\otimes y+L^{2}\right) \neq 0$, hence $\operatorname{dim} \operatorname{Im} \gamma_{3}^{\prime} \geq 1$. By using Theorem 2.6. we have $\operatorname{dim} \mathcal{M}(L) \leq 9$, which is a contradiction.

If $Z(L) \not \subset L^{2}$ then $t=\operatorname{dim} Z(L) /\left(Z(L) \cap L^{2}\right) \geq 1$. By using Theorem 2.5, we have $\operatorname{dim} \mathcal{M}(L) \leq 7$, which is a contradiction.

Hence the assumption is false and the result follows.

Theorem 3.6. There is no n-dimensional nilpotent Lie algebra of nilpotency class 3 such that $\operatorname{dim} \mathcal{M}(L)$ attains the bound.

Proof. By contrary, let there be a Lie algebra $L$ such that $\operatorname{dim} \mathcal{M}(L)$ attains the bound. Consider an ideal $I$ in $Z(L) \cap L^{2}$ of dimension one. By Proposition 3.3 $\operatorname{dim} \mathcal{M}(L / I)$ also attains the bound. If $\operatorname{cl}(L / I)=2$, then we have a contradiction by using Lemma 3.5. Now if $\operatorname{cl}(L / I)=3$, then we proceed by induction on $n$ to show that there is no such Lie algebra $L$ such that $\operatorname{dim} \mathcal{M}(L)$ attains the bound. For $n=5, L / I$ is of maximal class and so $\operatorname{dim}(L / I)^{2}=2$. Hence by using Lemma 3.1 and Proposition 3.3 there is no such a Lie algebras. So we have a contradiction. Let $n>5$. If $K / I$ is a one-dimensional ideal of $L / I$ is contained in $Z(L / I) \cap(L / I)^{2}$ such that $(L / I) /(K / I)$ is of class 2 , then the result follows from Proposition 3.3 and Lemma 3.5. Now consider $\operatorname{cl}((L / I) /(K / I))=3$. Since $\operatorname{dim} L / I=n-1$, by using the induction hypothesis there is no such a Lie algebra $L / I$ such that $\operatorname{dim} \mathcal{M}(L / I)$ attains the bound, which is contradiction.

Theorem 3.7. There is no n-dimensional nilpotent Lie algebra of nilpotency class $c \geq 3$ such that $\operatorname{dim} \mathcal{M}(L)$ attains the bound. That means, $\operatorname{dim} \mathcal{M}(L) \leq \frac{1}{2}(n-m-$ $1)(n+m)-\left(\sum_{i=2}^{\min \{n-m, c\}} n-m-i\right)-1$, for all Lie algebras of nilpotency class $c \geq 3$.

Proof. We proceed by using induction on $c$. If $c=3$ and $I$ be a one dimensional ideal in $L^{2} \cap Z(L)$. Then the result follows from Theorem 3.6. Now let $c>3$. If $c l(L / I)<c$, then by using the induction hypothesis on $c$, there is no $L / I$ such that $\operatorname{dim} \mathcal{M}(L / I)$ attains the bound. Hence the result follows by Proposition 3.3 Now let $\operatorname{cl}(L / I)=c$. If $K / I$ is a one dimensional ideal $L / I$ such that $K / I \subseteq$ $Z(L / I) \cap(L / I)^{2}$. Then, we use induction on $n$ to prove our result. Obviously $n \geq c+1$ and Lemma 3.1 emphasis $n>c+1$. If $n=c+2$, then $L / I$ is of maximal class. So the result is obtained by using $n=c+2$ by using Lemma 3.1 and Proposition 3.3. Let $n>c+2$. If $K / I$ is an ideal in $L / I$ of one dimensional such that $K / I \subseteq Z(L / I) \cap(L / I)^{2}$ and $\left.c l(L / I) /(K / I)\right)<c$ then the result follows by using the induction hypothesis on $c$ and by Proposition 3.3. Now if $c l((L / I) /(K / I)=c$. Since $\operatorname{dim} L / I=n-1$, by the induction hypothesis on $n$ there is no such Lie algebra $L / I$ that $\operatorname{dim} \mathcal{M}(L / I)$ attains the bound. Hence by using Proposition 3.3 is completes the proof.

Corollary 3.8. Let $L$ be a non-abelian nilpotent Lie algebra of class $c$. Then $\operatorname{dim} \mathcal{M}(L)$ attains the bound if and only if $L$ is isomorphic to one of the Lie algebras $H(1) \oplus A(n-3), L_{5,8}$ or $L_{6,26}$. 
Proof. The result follows from Theorems 3.2 and 3.7.

In the following examples, we may use method of Hardy and Stitzinger in 3 to show that there are some Lie algebras of dimension $n$ with the derived subalgebra of dimension $m$ such that $\operatorname{dim} \mathcal{M}(L)=\frac{1}{2}(n-m-1)(n+m)-\left(\sum_{i=2}^{\min \{n-m, c\}} n-m-i\right)-1$.

Example 3.9. Let $L \cong L_{5,7}=\left\langle x_{1}, \ldots, x_{5}\right|\left[x_{1}, x_{2}\right]=x_{3},\left[x_{1}, x_{3}\right]=x_{4},\left[x_{1}, x_{4}\right]=$ $\left.x_{5}\right\rangle$ or $L \cong L_{5,9}=\left\langle x_{1}, \ldots, x_{5} \mid\left[x_{1}, x_{2}\right]=x_{3},\left[x_{1}, x_{3}\right]=x_{4},\left[x_{2}, x_{3}\right]=x_{5}\right\rangle$.

By using the method of Hardy and Stitzinger in [3] we have $\operatorname{dim} \mathcal{M}\left(L_{5,7}\right)=$ $\operatorname{dim} \mathcal{M}\left(L_{5,9}\right)=3$. Therefore $L_{5,7}$ and $L_{5,9}$ obtain the upper bound mentioned in the Theorem 3.7

\section{REFERENCES}

[1] Ellis, G.,On the Schur multiplier of p-groups., Comm. Algebra, (1999), 27(9): 4173-4177.

[2] S. Hatui, Finite p-groups having Schur multiplier of maximal order, available at https://arxiv.org/abs/1610.07042.

[3] Hardy, P. and Stitzinger, E. ; On characterizing nilpotent Lie algebras by their multipliers, $\mathrm{t}(\mathrm{L})=3,4,5,6$. Comm. Algebra 26(1998), no. 11, 35273539.

[4] Lazard, M. ; Groupes analytiques p-adiques, Publ. IHES No. 26, (1965).

[5] Niroomand, P., Parvizi, M. and Russo, F.G.; Some criteria for detecting capable Lie algebras. J. Algebra 384 (2013), 3644.

[6] Niroomand, P. and Russo, F.G.; A note on the Schur multiplier of a nilpotent Lie algebra. Comm. Algebra 39 (2013), no. 4, 1293-1297.

[7] Niroomand, P. and Russo, F.G.; "A restriction on the Schur multiplier of nilpotent Lie algebras. (2011). 1081-3810.

[8] Niroomand, P., A note on the Schur multiplier of groups of prime power order, Ricerche di Matematica, 61 (2012), 341-346

[9] Niroomand, P. and Johari, F.; Some results on the Schur multiplier of nilpotent Lie algebras. (2017) arXiv preprint arXiv:1701.03956

[10] Niroomand, P., Johari, F. and Parvizi, M.; Capable Lie algebras with the derived subalgebra of dimension two over an arbitrary field. arXiv preprint arXiv:1712.01542 (2017).

[11] Rai, P. K. On classication of groups having Schur multiplier of maximum order, Arch. Math., (2016).

[12] Rai, P. K., On the dimension of the Schur multiplier of nilpotent Lie algberas. (2017) arXiv preprint arXiv: 1705.03208

[13] Saeedi, F; Arabyani, H. and Niroomand, P.; On dimension of Schur multiplier of nilpotent Lie algebras II. Asian-European Journal of Mathematics 10 (2016), no. 4, 1750076, 8pp.

[14] Schur, I, Uber die Darstellung der endlichen Gruppen durch gebrochene lineare Substitutionen, J. Reine Angew. Math, 127, (1904), 20-50.

[15] Salemkar. A. R., Alamian. V. and Mohammadzadeh. H.; Some properties of the Schur multiplier and covers of Lie algebras. Comm. Algebra 36 (2008), no. 2, 697707.

E-mail address: Shamsaki.Afsaneh@yahoo.com

E-mail address: p_niroomand@yahoo.com

DAMGHAN UNIVERSITY

E-mail address: Shamsaki.Afsaneh@yahoo.com 